\title{
Band structure of thin films by the linear augmented-plane-wave method
}

\author{
Jepsen, O.; Madsen, J.; Andersen, Ole Krogh
}

Published in:

Physical Review B

Link to article, DOI:

10.1103/PhysRevB.18.605

Publication date:

1978

Document Version

Publisher's PDF, also known as Version of record

Link back to DTU Orbit

Citation (APA):

Jepsen, O., Madsen, J., \& Andersen, O. K. (1978). Band structure of thin films by the linear augmented-planewave method. Physical Review B, 18(2), 605-615. https://doi.org/10.1103/PhysRevB.18.605

\section{General rights}

Copyright and moral rights for the publications made accessible in the public portal are retained by the authors and/or other copyright owners and it is a condition of accessing publications that users recognise and abide by the legal requirements associated with these rights.

- Users may download and print one copy of any publication from the public portal for the purpose of private study or research.

- You may not further distribute the material or use it for any profit-making activity or commercial gain

- You may freely distribute the URL identifying the publication in the public portal

If you believe that this document breaches copyright please contact us providing details, and we will remove access to the work immediately and investigate your claim. 


\title{
Band structure of thin films by the linear augmented-plane-wave method
}

\author{
O. Jepsen \\ Nordita, DK 2100 Copenhagen, Denmark \\ J. Madsen and O. K. Andersen \\ Electrophysics Department, Technical University, DK 2800 Lyngby, Denmark
}

(Received 19 December 1977)

\begin{abstract}
We present a linear augmented-plane-wave method for solving the band-structure problem in thin crystalline films. The potential is separated into a muffin-tin potential inside the film, a potential depending exclusively on the normal coordinate outside the film, and corrections in both regions. The method is tested on (100) and (111) monolayers of $\mathrm{Cu}$ using a standard muffin-tin potential.
\end{abstract}

\section{INTRODUCTION}

With the aim of studying chemisorbtion on transition-metal surfaces using the density-functional formalism ${ }^{1}$ we have developed an efficient selfconsistent band-structure method for thin films. Our method is an adaption of the linear augmented-plane-wave (LAPW) method $^{2-4}$ to the film geometry shown in Fig. 1. In the present paper we describe and test the film LAPW method for solving the band-structure problem and in subsequent papers we will treat the problems of selfconsistency and interatomic-force calculations.

Recent calculations of the binding in bulk transition metals ${ }^{5,6}$ and in diatomic molecules, ${ }^{7}$ including transition-metal dimers ${ }^{8}$ seem to indicate that the local approximation ${ }^{9}$ to the density-functional formalism will also be useful for studying chemical binding near transition-metal surfaces. The one-electron problem for a surface is tedious, however, because the symmetry is low, there are many inequivalent atoms, and the muffin-tin approximation is too crude for the potential.

The linear muffin-tin-orbital (LMTO) method, ${ }^{2,10}$ employed for the bulk and molecular calculations in Refs. 6-8, will undoubtedly prove useful for thin-film calculations. In fact, muffin-tin orbitals (MTO's) have for some time been used in nonself-consistent calculations for a geometry of alternating layers of film and vacuum, ${ }^{11}$ and efficient LMTO formalisms for thin films have recently been devised. ${ }^{12}$ The techniques of cellular integration $^{10}$ employed in Refs. 7, 8, and 11 may; however, prove inconvenient when calculating interatomic forces, because the cell shapes generally change as the atoms are displaced. One should probably abandon the cells and perform the integrals over the interstitial region between the muffin tins by a Fourier-transform technique similar to the one described in Sec. IV B of Ref. 2.

In this situation we have preferred to investigate the potential of the IAPW method as an alternative to the LMTO method, despite the fact that an adequate description of the wave functions in a transition-metal system requires 30-40 LAPW's per atom but only nine ( $s, p$, and $d$ ) MTO's. (This corresponds to $\mathrm{mRy}$ accuracy for the eigenvalues.) We have thus given higher priority to accuracy and ease of programming than to physical transparency and computational speed, but it is our hope that, once obtained, the results of thin-film LAPW calculations may be interpreted using a simple real-space description such as the one provided by the atomic-sphere approximation ${ }^{2,12,13}$ to the LMTO method.

In addition to those mentioned above, ${ }^{11,12}$ several methods ${ }^{14-17}$ have been proposed for calculating band structures of transition-metal thin films. The film Korringa-Kohn-Rostoker (KKR) method ${ }^{15}$ is equivalent to the film LMTO method ${ }^{12}$ applied to a muffin-tin potential but it does not have the advantage of leading to an eigenvalue problem. The self-consistent pseudopotential method of Louie et $a l .{ }^{16}$ makes use of the sandwich geometry mentioned above. ${ }^{11}$ Even in bulk Nb, this method needs at least 100 OPW's per atom to converge to mRy accuracy, and for transition metals at the end of a series convergence will presumably be even slower. In the self-consistent LCAO method of Gay et al.$^{17}$ the electron density outside the film is described by the tails of the atomic orbitals. Whether this approximation is adequate for the chemisorption problem remains to be seen. Moreover, the basis set must include at least all occupied atomic orbitals and this will make the application of the LCAO method to $4 d$ and $5 d$ transition metals rather costly. So far, only the two last-mentioned calculations have been carried to self-consistency and computations of total energies or interatomic forces, have not been attempted.

In Sec. II we review the ideas behind the linear one-electron methods, ${ }^{2}$ and in Sec. III we present the details of the LAPW formalism for thin films. 
The method is tested in Sec. IV by computing the energy bands and state densities for (100) and (111) monolayers of $\mathrm{Cu}$ and the results are compared with those of Kar and Soven. ${ }^{15}$ Our density-ofstates calculations were performed by the triangle method, which is analogous to the tetrahedron method $^{18}$ used for three-dimensional Brillouin zone integrations. This technique is described in Appendix A.

\section{LINEAR ONE-ELECTRON METHODS}

In a linear method ${ }^{2}$ the solutions $\Psi_{j}(\overrightarrow{\mathrm{r}})$ of Schrödinger's equation are approximated by linear combinations

$$
\psi_{j}(\overrightarrow{\mathrm{r}})=\sum_{\vec{G}} A_{\overrightarrow{\mathrm{G}} j} \psi_{\vec{G}}(\overrightarrow{\mathrm{r}})
$$

of energy-independent basis functions $\psi_{\vec{G}}(\vec{r})$. The coefficients $A_{\overrightarrow{\mathrm{G}} j}$ are determined from the RayleighRitz variational principle which leads to the eigenvalue equations

$$
\sum_{\overrightarrow{\mathrm{G}}}\left(H_{\overrightarrow{\mathrm{G}} \cdot \overrightarrow{\mathrm{G}}}-E_{j} O_{\overrightarrow{\mathrm{G}} \cdot \overrightarrow{\mathrm{G}}}\right) A_{\overrightarrow{\mathrm{G}} j}=0 .
$$

Here, $H_{\vec{G} \cdot \vec{G}} \equiv\left\langle\psi_{\vec{G} \cdot}|H| \psi_{\vec{G}}\right\rangle$ and $O_{\vec{G} \cdot \vec{G}} \equiv\left\langle\psi_{\vec{G} \cdot} \mid \psi_{\vec{G}}\right\rangle$ are the Hamiltonian and overlap matrices.

In the construction of $\psi_{\vec{G}}$, advantage is taken of the fact that, in those regions of space where the kinetic energy $E-v(\overrightarrow{\mathrm{r}})$ of the electron is numerically large and the wave function therefore varies rapidly, the one-electron potential $v(\overrightarrow{\mathbf{r}})$ essentially has the muffin-tin form. This is spherically symmetric inside nonoverlapping spheres surrounding the atoms and, for the film geometry shown in Fig. 1, it is independent of $\vec{r}_{\|}$in the vacuum regions outside a slab containing the spheres. In each muffin-tin (i.e., inside a sphere

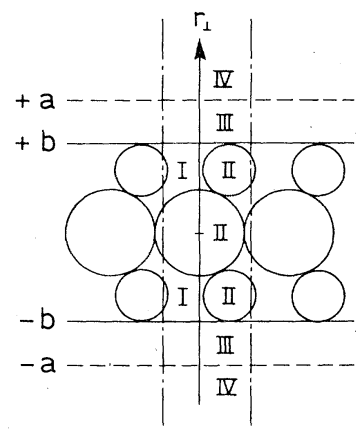

FIG. 1. Film geometry. The primitive cell of crosssectional area $A$ is indicated by the dashed-dot lines. Region I is the interstitial region, II are the spherical muffin-tin regions, and III + IV are the planar muffintin regions. or outside the slab) Schrödinger's differential equation $\left(H_{\overrightarrow{\mathrm{a}}}-E\right) \phi(E, \overrightarrow{\mathrm{q}})=0$ for the muffin-tin potential separates such that the solutions are of the form

$$
\phi_{L}(E, \vec{q})=\phi_{L}(E, q) F_{L}(\hat{q}) \text {. }
$$

Here, $\phi_{L}(E, q)$ is the regular solution of the onedimensional differential equation $\left(H_{L q}-E\right) \phi_{L}(E, q)$ $=0$, for quantum number $L$ and energy $E$, and $F_{L}(\hat{q})$ is a complete set of orthonormal functions spanning the boundary of the muffin tin.

Specificially, for a sphere centered at $\vec{Q}$ and of radius $s_{\vec{Q}}$ :

$$
\begin{aligned}
& q=|\overrightarrow{\mathrm{r}}-\overrightarrow{\mathrm{Q}}|, \quad 0 \leqslant q \leqslant s_{\overrightarrow{\mathrm{Q}}}, \\
& \hat{q}=(\overrightarrow{\mathrm{r}}-\overrightarrow{\mathrm{Q}}) /|\overrightarrow{\mathrm{r}}-\overrightarrow{\mathrm{Q}}|, \quad L=(\overrightarrow{\mathrm{Q}}, l, m), \\
& \phi_{L}(E, q)=\phi_{\overrightarrow{\mathrm{Q}} l}(E, q), \quad F_{L}(\hat{q})=i^{l} Y_{l m}(\hat{q}),
\end{aligned}
$$

and $\phi_{l}$ tends towards a constant times $q^{l}$ for small $q$. For a vacuum region, extending from $\vec{Q}$ $=Q_{\perp}(= \pm b)$ towards infinity:

$$
\begin{aligned}
& q=-\left|r_{\perp}-Q_{\perp}\right|, \quad-\infty<q \leqslant 0, \\
& \hat{q}=\overrightarrow{\mathrm{r}}_{\|}, \quad L=\left(Q_{\perp}, \overrightarrow{\mathrm{K}}_{\| 1}\right), \\
& \phi_{L}(E, q)=\phi_{Q_{\perp}} \overrightarrow{\mathrm{K}}_{\|}(E, q), \quad F_{L}(\hat{q})=A^{-1 / 2} e^{i \overrightarrow{\mathrm{K}}_{\| 1} \cdot \hat{\mathrm{q}}},
\end{aligned}
$$

and $\phi_{Q_{\perp} \vec{K}_{11}}$ tends towards a constant times $\exp \left[q\left(K_{\|}^{2}-E\right)^{1 / 2}\right]$ for $q$ tending towards minus infinity.

If the one-electron potential in the muffin tin at $\vec{Q}$ consists of the muffin-tin part only, i.e., it has the form $v_{\vec{Q}}(q)$, a solution $\Psi_{j}$ of Schrödinger's equation may be expressed as

$$
\Psi_{j}(\overrightarrow{\mathrm{r}})=\sum_{L} B_{L j} \phi_{L}\left(E_{j}, \overrightarrow{\mathrm{q}}\right)
$$

in that muffin tin.

The essential idea behind a linear method is based on the observation that the functions $\phi_{L}(E, q)$ are usually linear in energy over almost the entire range of interest, which covers of order one Rydberg. That is,

$$
\phi_{L}(E, q) \approx \phi_{L \nu}(q)+\left(E-E_{\nu}\right) \dot{\phi}_{L \nu}(q),
$$

where $E_{\nu}$ is an arbitrary energy in the middle of the range of interest and where $\phi_{L \nu}(q) \equiv \phi_{L}\left(E_{\nu}, q\right)$ and $\dot{\phi}_{L \nu}(q) \equiv\left[\partial \phi_{L}(E, q) / \partial E\right]_{E_{\nu}}$. With $\phi_{L}(E, \overrightarrow{\mathrm{q}})$ nor malized to unity in the appropriate muffin tin, $\phi_{L \nu}(\overrightarrow{\mathrm{q}})$ and $\dot{\phi}_{L \nu}(\overrightarrow{\mathrm{q}})$ will be orthogonal.

The basis functions in (1) are now defined as a set of functions which are continuous and differentiable in all space and are augmented as follows:

$$
\psi_{\vec{G}}(\overrightarrow{\mathrm{r}})=\sum_{L} C_{\overrightarrow{\mathrm{G}} L}\left[\phi_{L \nu}(\overrightarrow{\mathrm{q}})+\omega_{\overrightarrow{\mathrm{G}} L} \dot{\phi}_{L \nu}(\overrightarrow{\mathrm{q}})\right]
$$

in each muffin tin. It may be shown that $\phi_{L \nu}(q)$ 
and $\dot{\phi}_{L \nu}(q)$ have different logarithmic derivatives at the muffin tin [i.e., $\phi_{L \nu}^{\prime}(s) / \phi_{L \nu}(s) \neq \dot{\phi}_{L \nu}^{\prime}(s) / \dot{\phi}_{L \nu}(s)$ where, for a spherical tin, $s$ is the radius and, for a planar tin, $s$ is zero] and the conditions of continuity and differentiability therefore uniquely determine the coefficients $C_{\overrightarrow{\mathrm{G}} L}$ and $\omega_{\overrightarrow{\mathrm{G}} L}$.

In the film LAPW method the basis functions in the interstitial region are the set of symmetrized plane waves which vanish at $r_{1}= \pm a$. Since this set is complete in the entire $a$ slab (see Fig. 1), it is overcomplete in the interstitial region, and it is this degree of freedom which is administered by the variational principle in such a way that for a solution of (2),

$$
\sum_{\overrightarrow{\mathrm{G}}} A_{\overrightarrow{\mathrm{G}} j} C_{\overrightarrow{\mathrm{G}} L} \omega_{\overrightarrow{\mathrm{G}} L} / \sum_{\overrightarrow{\mathrm{G}}} A_{\overrightarrow{\mathrm{G}}_{j}} C_{\overrightarrow{\mathrm{G}}_{L} L} \approx E_{j}-E_{\nu}
$$

for all $L$, as we would expect by comparison of (1) and (8) with (6) and (7). Since the error of (7)is of order $\left(E-E_{\nu}\right)^{2}$, we expect that the errors of the eigenvalues obtained from (2) are of order $\left(E_{j}-E_{\nu}\right)^{4}$. This, as well as (9), is only true when the Hamiltonian in (2) has the muffin-tin form, i.e., $v(\overrightarrow{\mathbf{r}})=v_{\vec{Q}}(q)$ in all muffin tins. If the potential has non-muffin-tin contributions inside the muffin tins, the exact eigenfunctions cannot be written as in (6), and the eigenvalues obtained from the variational principle will, consequently, have additional errors of second order in the non-muffintin contributions. It may be noted that, in principle, there are no errors from the interstitial region.

\section{FILM LAPW FORMALISM}

We consider a film periodic in the $\overrightarrow{\mathbf{r}}_{11}$ direction as indicated in Fig. 1. In order to be able to split the eigenvalue problem for a given Bloch vector $\vec{k}_{\| 1}$ into even and odd parts, we shall restrict ourselves to considering films with reflection symmetry about the $r_{\perp}=0$ plane, where $r_{\perp}$ is the coordinate perpendicular to $\overrightarrow{\mathrm{r}}_{11}$. The translation vectors are $\vec{R}_{\| 1}$, the primitive-cell area is $A$, and the reciprocal lattice vectors are $\vec{G}_{11}$. The threedimensional vector $\left(\vec{r}_{\|}, r_{\perp}\right)$ is $\vec{r}$. We express the total one-electron potential as

$$
v(\overrightarrow{\mathbf{r}})=\sum_{\overrightarrow{\mathrm{Q}}} v_{\overrightarrow{\mathrm{Q}}}(q)+V_{\mathrm{mtz}}+v_{\mathrm{nmt}}(\overrightarrow{\mathrm{r}})
$$

with $\vec{Q}$ denoting the positions of the muffin tins, and with $\ddot{q}$ being defined as in (4) and (5). The potentials $v_{\vec{Q}}$ vanish outside their respective muffin tins, and $V_{\mathrm{mtz}}$ is constant in the interstitial region (I) and vanishes everywhere else. The remaining non-muffin-tin part of the potential is $v_{\text {nmt }}$, which we shall assume vanishes outside the $a$ slab. In what follows we shall closely follow the notation of Ref. 2, which we denote as linear methods (LM).

\section{A. Interstitial region (I)}

In the interstitial region, the LAPW's are defined as being the even and odd plane waves

$$
\begin{aligned}
\chi_{\overrightarrow{\mathrm{K}}}(\overrightarrow{\mathrm{r}}) & =\left(\frac{2}{\Omega}\right)^{1 / 2} e^{i \overrightarrow{\mathrm{K}}_{\| 1} \cdot \overrightarrow{\mathrm{r}}_{\| 1}} \times\left\{\begin{array}{l}
\cos G_{\perp} r_{\perp} \\
\sin G_{\perp} r_{\perp}
\end{array}\right. \\
& =\left\{\begin{array}{l}
(2 / \Omega)^{1 / 2} \times \frac{1}{2}\left(e^{i \overrightarrow{\mathrm{K}}_{+} \cdot \overrightarrow{\mathrm{r}}}+e^{i \overrightarrow{\mathrm{K}}_{-} \cdot \overrightarrow{\mathrm{r}}}\right), \\
(2 / \Omega)^{1 / 2} \times \frac{1}{2}(-i)\left(e^{i \overrightarrow{\mathrm{K}}_{+} \cdot \overrightarrow{\mathrm{r}}}-e^{i \overrightarrow{\mathrm{K}}_{-} \cdot \overrightarrow{\mathrm{r}}}\right),
\end{array}\right.
\end{aligned}
$$

where $\Omega=2 a A$ and

$$
\begin{aligned}
& \overrightarrow{\mathrm{K}}_{11} \equiv \overrightarrow{\mathrm{K}}_{11}+\overrightarrow{\mathrm{G}}_{11}, \quad \overrightarrow{\mathrm{K}}_{+} \equiv \overrightarrow{\mathrm{K}}_{\| 1}+\overrightarrow{\mathrm{G}}_{\perp} \equiv \overrightarrow{\mathrm{K}}, \\
& \overrightarrow{\mathrm{K}}_{-} \equiv \overrightarrow{\mathrm{K}}_{11}-\overrightarrow{\mathrm{G}}_{\perp}, \quad K=K_{+}=K_{-}=\left(K_{11}^{2}+G_{\perp}^{2}\right)^{1 / 2}, \\
& \overrightarrow{\mathrm{G}} \equiv \overrightarrow{\mathrm{G}}_{\| 1}+\overrightarrow{\mathrm{G}}_{\perp} .
\end{aligned}
$$

Furthermore,

$$
G_{\perp}=n \pi / 2 a,
$$

where

$$
n=\left\{\begin{array}{l}
1,3,5, \cdots, \\
2,4,6, \cdots,
\end{array}\right.
$$

such that the set $\chi_{\overrightarrow{\mathrm{K}}}$ is complete and orthonormal in the $a$ slab, and each function vanishes for $r_{\perp}$ $=a$ and $-a$. Throughout this paper we shall adopt the notation that the upper (lower) expression or sign corresponds to the even (odd) parity.

\section{B. Spherical muffin tins (II)}

Inside any sphere, the LAPW is that linear combination of the functions

$$
\Phi_{\overrightarrow{\mathrm{Q}} \imath m}(D, \overrightarrow{\mathrm{q}})=\phi_{\overrightarrow{\mathrm{Q}} l m \nu}(\overrightarrow{\mathrm{q}})+\omega_{\overrightarrow{\mathrm{Q}} \imath}(D) \dot{\phi}_{\overrightarrow{\mathrm{Q}} l m \nu}(\overrightarrow{\mathrm{q}}),
$$

defined in (LM2.2), (LM2.3), and (LM2.7), ${ }^{2}$ which matches continuously and differentiably onto the function (11). Therefore, in the sphere surrounding the atom at $\vec{Q}$,

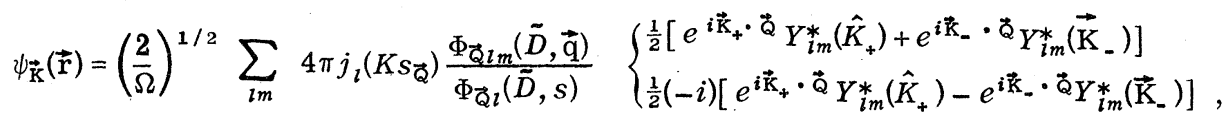


where $\tilde{D} \equiv \tilde{D}_{\vec{Q} l}\left(K s_{\vec{Q}}\right)$ is the free-electron logarithmic derivative given by (LM 2.29).

\section{Planar muffin tins $(I I I+I V)$}

Since vacuum regions or planar muffin tins are not specifically dealt with in LM, we shall now consider them in some detail.

Inside a planar muffin tin, the solution of Schrödinger's differential equation for the muffin-tin potential and the energy $E$ is

$$
\phi_{\overrightarrow{\mathrm{K}}_{\| 1}}(E, \overrightarrow{\mathrm{q}})=A^{-1 / 2} e^{i \overrightarrow{\mathrm{K}}_{\| 1} \cdot \overrightarrow{\mathrm{r}}_{11}} \phi(\mathcal{E}, q) .
$$

Here, $\phi(\mathscr{E}, q)$ depends on the energy, the Bloch vector, and the reciprocal-lattice vector through

$$
\mathcal{E}=E-\left|\overrightarrow{\mathrm{k}}_{\|}+\overrightarrow{\mathrm{G}}_{\|}\right|^{2}=E-K_{\|}^{2},
$$

where $\mathcal{E}$ is assumed to fall below $v(-\infty)$, and $\phi(\mathscr{E}, q)$ satisfies the one-dimensional Schrödinger equation

$$
\left(\frac{-\partial^{2}}{\partial q^{2}}+v(q)-\mathcal{E}\right) \phi(\mathcal{E}, q)=0,
$$

The normalization is

$$
\left\langle\phi^{2}(\mathcal{E}, q)\right\rangle=\int_{-\infty}^{0} \phi^{2}(\mathcal{E}, q) d q=1 .
$$

For each value of $\vec{G}_{\|}$we use the energy- and Bloch-vector-independent functions

$$
\phi_{\nu}(q) \equiv \phi\left(\mathcal{E}_{\nu}, q\right) \text { and } \dot{\phi}_{\nu}(q) \equiv\left(\frac{\partial \phi(\mathcal{E}, q)}{\partial \mathcal{E}}\right)_{\mathcal{E}=\mathcal{E}_{\nu}},
$$

where, instead of fixing $E_{\nu}$ and using $\mathcal{E}_{\nu}\left(\overrightarrow{\mathrm{k}}_{\|}, \overrightarrow{\mathrm{G}}_{\|}\right)$, we fix

$$
\mathcal{E}_{\nu}=\mathcal{E}_{\nu}\left(\vec{G}_{\| 1}\right)
$$

and hence use the linear expansion (24) to express the $\overrightarrow{\mathrm{k}}_{11}$ as well as the $E$ dependence of the wave function in the vacuum regions.

As a consequence of $(19), \phi_{\nu}(q)$ and $\dot{\phi}_{\nu}(q)$ are orthogonal. The outgoing logarithmic derivatives at the boundary of the planar muffin tin are

$$
D_{\nu}=\phi_{\nu}^{\prime} / \phi_{\nu} \text { and } D_{\nu}^{1}=\dot{\phi}_{\nu}^{\prime} / \dot{\phi}_{\nu}
$$

and these are always different because

$$
\left(D_{\nu}-D_{\nu}^{1}\right) \phi_{\nu} \dot{\phi}_{\nu}=1 \text {. }
$$

We have adopted the notation $\phi_{\nu} \equiv \phi_{\nu}(0)$ and $\dot{\phi}_{\nu}$ $\equiv \dot{\phi}_{\nu}(0)$. From $\phi_{\nu}(q)$ and $\dot{\phi}_{\nu}(q)$ we can now construct a function of arbitrary logaxithmic derivative $D$, that is

$$
\Phi(D, q)=\phi_{\nu}(q)+\omega(D) \dot{\phi}_{\nu}(q),
$$

where

$$
\omega(D)=-\frac{\phi_{\nu}}{\dot{\phi}_{\nu}} \frac{D-D_{\nu}}{D-D_{\nu}^{1}}=\phi_{\nu}^{2}\left(D_{\nu}^{1}-D_{\nu}\right) \frac{D-D_{\nu}}{D-D_{\nu}^{1}} .
$$

In the basis of

$$
\Phi_{\overrightarrow{\mathrm{K}}_{\| \mid}}(D, \overrightarrow{\mathrm{q}})=A^{-1 / 2} e^{i \overrightarrow{\mathrm{K}}_{\| \mid}} \cdot \overrightarrow{\mathrm{r}}_{\mid 1} \Phi(D, q),
$$

the matrix elements of the Hamiltonian $H_{\vec{a}}$ $\equiv-\nabla^{2}+v(q)$ for a planar muffin tin are then

$$
\begin{aligned}
& \left\langle\Phi_{\overrightarrow{\mathrm{K}}_{\|}^{\prime}}\left(D^{\prime}\right)\left|\mathrm{H}_{\overrightarrow{\mathrm{q}}}\right| \Phi_{\overrightarrow{\mathrm{K}}_{\| 1}}(D)\right\rangle \\
& \quad=\delta_{\overrightarrow{\mathrm{G}}_{i \|}, \overrightarrow{\mathrm{G}}_{\| \mid}}\left\{\omega(D)+\left(\mathcal{E}_{\nu}+K_{\|}^{2}\right)\left[1+\omega\left(D^{\prime}\right) \omega(D)\left\langle\dot{\phi}_{\nu}^{2}\right\rangle\right]\right\},
\end{aligned}
$$

where $\overrightarrow{\mathrm{K}}_{\|}^{\prime}=\overrightarrow{\mathrm{k}}_{\|}+\overrightarrow{\mathrm{G}}_{\|}^{\prime}$ and $\overrightarrow{\mathrm{K}}_{\|}=\overrightarrow{\mathrm{k}}_{\|}+\overrightarrow{\mathrm{G}}_{\|}$.

The overlap integrals are

$$
\left\langle\Phi_{\overrightarrow{\mathrm{K}}_{\| 1}^{e}}\left(D^{\prime}\right) \mid \Phi_{\overrightarrow{\mathrm{K}}_{\| 1}}(D)\right\rangle=\delta_{\overrightarrow{\mathrm{G}}_{\| 1}^{o}, \overrightarrow{\mathrm{G}}_{\| 1}}\left[1+\omega\left(D^{\prime}\right) \omega(D)\left\langle\dot{\phi}_{\nu}^{2}\right\rangle\right] \text {. }
$$

Except for the Bloch vector dependence of $\mathcal{E}$, the formalism given above for a planar muffin tin is seen to be quite analogous to the one presented in LM for a spherical muffin tin.

In Fig. 2 we show $\phi_{\nu}^{2}, D_{\nu}, D_{\nu}^{1}$, and $\left\langle\dot{\phi}_{\nu}^{2}\right\rangle^{-1 / 2}$ as functions of the energy $\mathcal{E}_{\nu}$ for self-consistent potential ${ }^{9}$ outside a jellium film of width 5 a.u. and of density corresponding to $r_{s}=3 \mathrm{Bohr}$ radii. ${ }^{19}$ The variational estimate

$$
\mathcal{E}(D)=\mathcal{E}_{\nu}+\omega(D)\left[1+\omega^{2}(D)\left\langle\dot{\phi}_{\nu}^{2}\right\rangle\right]^{-1},
$$

of the function inverse to the logarithmic-derivative function, $D(\mathscr{E})=\phi^{\prime}(\mathcal{E}, 0) / \phi(\mathcal{E}, 0)$, is confined to the energy range $\mathcal{E}_{\nu} \pm \frac{1}{2}\left\langle\dot{\phi}_{\nu}^{2}\right\rangle^{-1 / 2}$ which, as seen from the figure, increases for increasing $\left|\mathcal{E}_{\nu}\right|$. For typical lattice sizes (see Sec. IV) the potential parameters for a given $\mathcal{E}_{\nu} \lesssim-0.5 \mathrm{Ry}$ apply for all

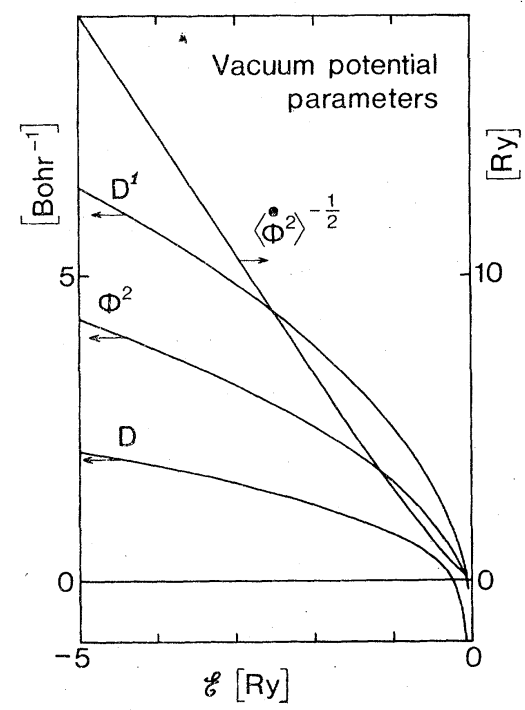

FIG. 2. Potential parameters as functions of $\mathcal{E}$ for the self-consistent potential (Ref. 9) outside a jellium film of width $5 \mathrm{bohr}$ radii and density corresponding to $r_{s}=3$ bohr radii (Ref, 19). 
Bloch vectors in the irreducible part of the twodimensional Brillouin zone, and for $\mathscr{E}_{\nu} \lessgtr-2$ Ry they also apply for several reciprocal lattice vectors. The potential parameters for a constant potential are given in Appendix B.

In a planar muffin tin the LAPW is given by (26), although normalized, and with $D$ chosen in such a way that (26) matches continuously and differentiably onto (11), i.e.,

$$
\psi_{\overrightarrow{\mathrm{k}}}(\overrightarrow{\mathrm{r}})=a^{-1 / 2} \frac{\Phi_{\overrightarrow{\mathrm{k}} \|}(\tilde{D}, \overrightarrow{\mathrm{q}})}{\Phi(\tilde{D}, 0)} \times\left\{\begin{array}{l}
\cos \left(G_{\perp} Q_{\perp}\right), \\
\sin \left(G_{\perp} Q_{\perp}\right),
\end{array}\right.
$$

where $Q_{1}= \pm b$ and

$$
\tilde{D}=\left\{\begin{array}{l}
G_{\perp} \tan \left(G_{\perp} Q_{\perp}\right), \\
-G_{\perp} \cot \left(G_{\perp} Q_{\perp}\right)
\end{array}\right.
$$

\section{Hamiltonian and overlap matrices}

For the one-electron potential (10), and with the LAPW representation defined by (11), (15), and (30), the Hamiltonian and overlap matrices may be expressed as

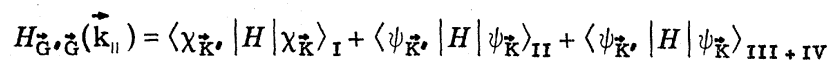

$$
\begin{aligned}
& =\left\langle\chi_{\overrightarrow{\mathrm{K}}} \cdot\left|-\nabla^{2}+V_{\mathrm{mtz}}\right| \chi_{\overrightarrow{\mathrm{K}}}\right\rangle_{\mathrm{I}+\mathrm{II}+\mathrm{III}}+\left\langle\psi_{\overrightarrow{\mathrm{K}}} \cdot\left|v_{\mathrm{nmt}}\right| \psi_{\overrightarrow{\mathrm{K}}}\right\rangle_{\mathrm{I}+\mathrm{II}+\mathrm{III}} \\
& +\left(\left\langle\psi_{\overrightarrow{\mathrm{K}}},\left|-\nabla^{2}+\sum v_{\stackrel{Q}{Q}}\right| \psi_{\overrightarrow{\mathrm{K}}}\right\rangle_{\mathrm{II}}-\left\langle\chi_{\overrightarrow{\mathrm{K}}^{\circ}}\left|-\nabla^{2}+V_{\mathrm{mtz}}\right| \chi_{\overrightarrow{\mathrm{R}}}\right\rangle_{\mathrm{II}}\right) \\
& +\left(\left\langle\psi_{\mathrm{K}^{\circ}}\left|-\nabla^{2}+\sum v_{\vec{Q}}\right| \psi_{\overrightarrow{\mathrm{K}}}\right\rangle_{\mathrm{III}+\mathrm{IV}}-\left\langle\chi_{\overrightarrow{\mathrm{K}}^{\prime}}\left|-\nabla^{2}+V_{\mathrm{mtz}}\right| \psi_{\overrightarrow{\mathrm{K}}}\right\rangle_{\mathrm{III}+\mathrm{IV}}\right. \\
& =\delta_{\overrightarrow{\mathrm{G}} \cdot \overrightarrow{\mathrm{G}}}\left(K^{2}+V_{\mathrm{mtz}}\right)+\left(\left\langle\psi_{\overrightarrow{\mathrm{K}}},\left|v_{\mathrm{nmt}}\right| \psi_{\overrightarrow{\mathrm{K}}}\right\rangle_{\mathrm{I}+\mathrm{II}+\mathrm{III}}\right. \\
& +\left(\sum_{\vec{Q} \in \mathbf{I I}} e^{i\left(\overrightarrow{\mathrm{G}}_{\| 1}-\overrightarrow{\mathrm{G}}_{\| 1}\right) \cdot \vec{Q}_{\| 1}} \sum_{l} W_{\vec{Q} l}\left(\overrightarrow{\mathrm{K}}^{\prime}, \overrightarrow{\mathrm{K}}\right)\left[\Gamma_{\vec{Q} l}\left(K^{\prime}, K\right)+E_{\nu} \Delta_{\vec{Q} l}\left(K^{\prime}, K\right)-V_{\mathrm{mtz}}\right]\right) \\
& +\delta_{\vec{G}_{\| 1}^{\prime} \vec{G}_{\| 1}} a^{-1}\left(B(0,0 ; 1) D_{\nu}-B\left(G_{\perp}^{\prime}, G_{\perp} ; D_{\nu}\right) \phi_{\nu} \dot{\phi}_{\nu}+\left(\mathcal{E}_{\nu}+K_{\| 1}^{2}\right)\left[B\left(G_{\perp}^{\prime}, G_{\perp} ; D_{\nu}^{1}\right) \dot{\phi}_{\nu}^{2}+B\left(G_{\perp}^{\prime}, G_{\perp} ; D_{\nu}\right) \phi_{\nu}^{2}\left\langle\dot{\phi}_{\nu}^{2}\right\rangle\right]\right. \\
& +\left(K_{11}^{2}+G_{\perp}^{\prime} G_{\perp}+V_{\mathrm{mtz}}\right)\left\{\left(G_{\perp}-G_{\perp}^{\prime}\right)^{-1} \sin \left[\left(G_{\perp}-G_{\perp}^{\prime}\right) b\right]-\delta_{G_{\perp}^{\prime} G_{\perp}} a\right\} \\
& \left. \pm\left(K_{\mathrm{u}}^{2}-G_{\perp}^{\prime} G_{\perp}+V_{\mathrm{mtz}}\right)\left(G_{\perp}+G_{\perp}^{\prime}\right)^{-1} \sin \left[\left(G_{\perp}+G_{\perp}^{\prime}\right) b\right]\right)
\end{aligned}
$$

and

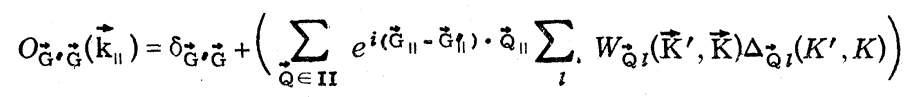

$$
\begin{aligned}
& +\delta_{\mathrm{G}_{\| 1}^{\prime}}^{*} \ddot{\mathrm{G}}_{11} a^{-1}\left\{B\left(G_{\perp}^{\prime}, G_{\perp} ; D_{\nu}^{1}\right) \dot{\phi}_{\nu}^{2}+B\left(G_{\perp}^{\prime}, G_{\perp} ; D_{\nu}\right) \phi_{\nu}^{2}\left\langle\dot{\phi}_{\nu}^{2}\right\rangle+\left(G_{\perp}-G_{\perp}^{\prime}\right)^{-1} \sin \left[\left(G_{\perp}-G_{\perp}^{\prime}\right) b\right]-\delta_{G_{\perp}^{\prime} G_{\perp}} a\right. \\
& \left. \pm\left(G_{\perp}+G_{\perp}^{\prime}\right)^{-1} \sin \left[\left(G_{\perp}+G_{\perp}^{\prime}\right) b\right]\right\} .
\end{aligned}
$$

In the contributions from the spherical muffin tins, each term in the $l$ sums is Hermitian, and the sums are converged at $l \approx 2-3$ provided that $V_{\mathrm{mtz}} \approx v_{\vec{Q}}\left(\mathrm{~s}_{\mathbb{Q}}\right)$ for all $\bar{Q}$. The functions $\Gamma$ and $\Delta$ are defined in (LM3.11)-(LM3.14) and $W$ is defined as in (LM3.4) with the Legendre polynomial $P_{l}\left(\hat{K}^{\prime} \cdot \hat{K}\right)$ substituted by

$$
\begin{aligned}
P_{l}\left(\overrightarrow{\mathrm{K}}^{\prime}, \overrightarrow{\mathrm{K}}\right)= & P_{\imath}\left(\hat{K}_{+}^{\prime} \cdot \hat{K}_{+}\right) \cos \left[\left(G_{\perp}-G_{\perp}^{\prime}\right) Q_{\perp}\right) \\
& \pm P_{l}\left(\hat{K}_{+}^{\prime} \cdot \hat{K}_{-}\right) \cos \left[\left(G_{\perp}+G_{\perp}^{\prime}\right) Q_{\perp}\right] .
\end{aligned}
$$

In the contributions from the vacuum regions

$$
B\left(G_{\perp}^{\prime}, G_{\perp} ; D\right) \equiv 2\left\{\begin{array}{l}
\left(G_{\perp}^{\prime} s^{\prime}-D c^{\prime}\right)\left(G_{\perp} s-D c\right) \\
\left(G_{\perp}^{\prime} c^{\prime}+D s^{\prime}\right)\left(G_{\perp} c+D s\right)
\end{array}\right.
$$

with $s \equiv \sin \left(b G_{\perp}\right), s^{\prime} \equiv \sin \left(b G_{\perp}^{\prime}\right), c \equiv \cos \left(b G_{\perp}\right)$, and $c^{\prime} \equiv \cos \left(b G_{\perp}^{\prime}\right)$.

Usually the non-muffin-tin part of the potential $v_{\text {nmt }}(\overrightarrow{\mathrm{r}})$ is significant only in the interstitial region and in the outer parts $\left(q \approx s_{\vec{Q}}\right.$ for spherical tins, and $q \approx 0$ for planar tins) of the muffin tins. In the interstitial region, the LAPW's equal the plane waves (11) and at the muffin-tin boundaries they are continuous and differentiable. Therefore, in the regions where $v_{\text {nmt }}$ is significant, the LAPW's resemble plane waves and the approximation

$$
\begin{aligned}
\left\langle\psi_{\overrightarrow{\mathrm{K}}^{\prime}} \cdot\left|v_{\mathrm{nmt}}\right| \psi_{\mathrm{R}_{\mathrm{K}}}\right\rangle_{\mathrm{I}+\mathrm{II}+\mathrm{III}} & \approx\left\langle\chi_{\mathrm{k}^{\prime}}\left|v_{\mathrm{nmt}}\right| \chi_{\overrightarrow{\mathrm{K}}}\right\rangle_{\mathrm{I}+\mathrm{II}+\mathrm{III}} \\
& \equiv v_{\mathrm{nmt}}(\overrightarrow{\mathrm{G}}, \overrightarrow{\mathrm{G}})
\end{aligned}
$$

is usually a good one. 


\section{RESULTS}

We first tested the film LAPW formalism on a square-well potential which was 1 Ry lower in the $b$ slab than elsewhere, and which had a width equal to that of the $\mathrm{Cu}$ films described below (Table I). The agreement with the exact results was perfect within the accuracy of our calculations. We now present and discuss results obtained for (100) and and (111) monolayers of fcc $\mathrm{Cu}$.

The primitive reciprocal lattice vectors for the (100) layer are $\overrightarrow{\mathrm{G}}_{\|}^{1}=(1,0)$ and $\overrightarrow{\mathrm{G}}_{\|}^{2}=(0,1)$, expressed in a cubic frame and in units of $(2 \sqrt{2}) \pi / a_{c}$, where $a_{c}$ is the lattice constant for the bulk. The corners of the irreducible part of the two-dimensional Brillouin zone (2D-IBZ) are $\vec{\Gamma}=(0,0), \vec{X}=\left(\frac{1}{2}, 0\right)$, and $\overrightarrow{\mathrm{M}}=\left(\frac{1}{2}, \frac{1}{2}\right)$. For the (111) layer, $\overrightarrow{\mathrm{G}}_{\| 1}^{1}=(1, \sqrt{3})$ and $\overrightarrow{\mathrm{G}}_{\|}^{2}=(2,0)$, in units of $(2 \sqrt{2}) \pi / a_{c} \sqrt{3}$, and the corners of the $2 \mathrm{D}-\mathrm{IBZ}$ are $\vec{\Gamma}=(0,0), \overrightarrow{\mathrm{M}}=(1,0)$, and $\overrightarrow{\mathrm{K}}=(1,1 / \sqrt{3})$.

We used a muffin-tin potential which, inside the $b$ slab (I + II), was the standard ${ }^{20}$ bulk potential for $\mathrm{Cu}$, obtained by superposing relativistic atomic charge densities on a bulk crystal and using Slater exchange with $\alpha=1$. In the vacuum region (III + IV) our potential was set equal to the self-consistent ${ }^{19}$ potential $^{9}$ outside a jellium film of the same width and of density corresponding to $r_{s}=3 \mathrm{Bohr}$ radii, the value appropriate for bulk $\mathrm{Cu} .^{21}$ Finally, the potential inside the $b$ slab was shifted rigidly upwards such that, for the (100) film, our $d$ bands fall in the same energy range as those of Kar and Soven. ${ }^{15}$ These authors superposed atomic charge densities on the proper monolayer and used $\alpha$ $=0.707$. Our potential parameters, which apply to the (100) as well as to the (111) film, may be found in Table I.

The summation over angular momenta in (32) and (33) is rapidly convergent, and we found that the errors of the energy eigenvalues caused by truncation after $l=2$ and 3 were less than 4 and $0.2 \mathrm{mRy}$, respectively. In the further calculations we included all terms with $l \leqslant 3$.

In Fig. 3 we illustrate the convergence of the energies as the number of LAPW's included in the basis set, and the "extra" width $a-b$ are increased. For a given value of $\max (K)$ (denoted $K$ in the figure) all LAPW's of a given parity, and with $\left|\vec{k}_{11}+\vec{G}_{\| 1}+\vec{G}_{\perp}\right| \leqslant \max (K)$, are included in the basis set. Of the two states considered in the figure, the energy of the odd state converges slower than that of the even state because the odd state

TABLE I. Potential parameters from which the band structures of the (100) and (111) monolayers of $\mathrm{Cu}$ were calculated. ${ }^{\mathrm{a}}$

\begin{tabular}{|c|c|c|c|c|c|c|}
\hline & & & $\overrightarrow{\mathrm{G}}_{\| 1}(1)$ & $\overrightarrow{\mathrm{G}}_{\|}(2)$ & $\overrightarrow{\mathrm{G}}_{\|}(3)$ & \\
\hline \multirow[t]{6}{*}{ Planar MT } & $\mathcal{E}_{\nu}-v(-\infty)$ & (Ry) & -1.0 & -2.0 & -5.0 & \\
\hline & $\phi_{\nu}^{2}$ & $\left(\right.$ bohr $\left.^{-1}\right)$ & 1.625 & 2.542 & 4.278 & \\
\hline & $D_{\nu}$ & $\left(\right.$ bohr $\left.^{-1}\right)$ & 0.770 & 1.251 & 2.132 & \\
\hline & $D_{\nu}^{1}$ & $\left(b^{\prime} h r^{-1}\right)$ & 2.527 & 3.865 & 6.446 & \\
\hline & $\left\langle\dot{\phi}_{\nu}^{2}\right\rangle^{-\frac{1}{2}}$ & (Ry) & 3.034 & 6.800 & 18.552 & \\
\hline & & & $s$ & $p$ & $d$ & $f$ \\
\hline \multirow[t]{7}{*}{ Spherical MT } & $E_{\nu}-V_{\mathrm{mtz}}$ & (Ry) & 0.500 & 0.500 & 0.500 & 0.500 \\
\hline & $\omega(-\ell-1)$ & (Ry) & -0.058 & 1.146 & -0.073 & 5.202 \\
\hline & $10 S \Phi^{2}(-\ell-1)$ & (Ry) & 5.204 & 4.803 & 0.307 & 5.749 \\
\hline & $\frac{\Phi(-\ell-1)}{\Phi(\ell)}$ & & 0.844 & 0.677 & -0.065 & 0.563 \\
\hline & $\left\langle\dot{\phi}_{v}^{2}\right\rangle^{-\frac{1}{2}}$ & (Ry) & 5.322 & 7.486 & 0.790 & 14.855 \\
\hline & $V_{\mathrm{mtz}}-v(-\infty)$ & (Ry) & & \multicolumn{2}{|c|}{-1.072} & \\
\hline & $\begin{array}{l}a_{c} \\
s=b=a_{c} / 2 \sqrt{2}\end{array}$ & (bohr) & & \multicolumn{2}{|c|}{6.831} & \\
\hline
\end{tabular}

\footnotetext{
${ }^{a}$ The three groups of reciprocal-lattice vectors are specified in Table II.
} 


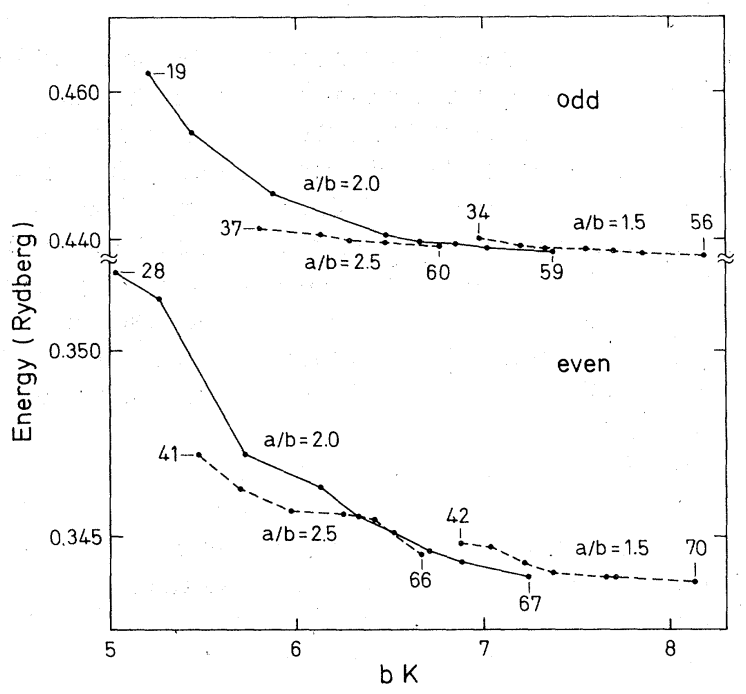

FIG. 3. Convergence of two of the eigenvalues for a single (100) layer of copper at $\overrightarrow{\mathrm{k}}_{\|}=\left(\frac{1}{3}, \frac{1}{6}\right)(2 \sqrt{2}) \pi / a_{c}$ as functions of $a / b$ (see Fig. 1) and the number of LAPW's included in the basis set. $K$ is the maximum value of $\left|\overrightarrow{\mathrm{k}}_{\|}+\overrightarrow{\mathrm{G}}_{\|} \pm \overrightarrow{\mathrm{G}}_{\perp}\right|$. The number of LAPW's, i.e., the dimension of the eigenvalue problem, is indicated for the smallest and largest $K$ for each value of $a / b$. For the single layer, $b$ equals the muffin-tin radius. The oddand even-parity states considered here have $d$ and $s p d$ character, respectively. Note the difference in the two energy scales.

is $d$ like and the even state is $s p d$ like. For $a-b$ $=0$ the trial function (1), constructed from any finite number of LAPW's, is forced to have nodes at the boundaries of the $b$ slab while, for $a-b>0$, there are no such constraints. The accuracy of the energies therefore increases with increasing values of $a$, provided that $b$ and $\max (K)$ are fixed, but the number of $G_{\perp}$ values also increases [see (13)]. For a given number of LAPW's (i.e., $\vec{G}$ vectors) above $30-40$, the accuracy of the energies is seen to be fairly independent of $(a-b)$ for values between 0.5 and 1.5 times the muffin-tin radius (i.e., $1.5 \leqslant a / b=a / s \leqslant 2.5$ ), which is the range of non-muffin-tin contributions in vacuum. ${ }^{22}$ The energies for the (111) layer converged in a way similar to what is shown in Fig. 3 , and we conclude that with about 30 odd and 35 even LAPW's, the energies are converged to better than $5 \mathrm{mRy}$. In the calculations to be described below, we set $(a-b)$ equal to the muffin-tin radius (i.e., $a / b$ $=a / s=2)$ and included 41 odd and 51 even LAPW's for the (100) film and 40 odd and 52 even LAPW's for the (111) film. This corresponds to $\max (s K)$ $\approx 6.5$, a value often employed in APW calculations for bulk transition metals.

In order to justify our use of $\vec{k}_{\| 1}$-independent potential parameters (21) for the planar muffin tins, and in order to minimize further the number of these parameters, we investigated the dependence of the energies on the choice of $\mathcal{E}_{\nu}=\mathcal{E}_{\nu}\left(\overrightarrow{\mathrm{G}}_{11}\right)$. Some results are given in Table II. For $\mathcal{E}_{\nu}$ less than about $-5 \mathrm{Ry},\left\langle\dot{\phi}_{\nu}^{2}\right\rangle^{-1 / 2}$ is larger than $20 \mathrm{Ry}$ and, using (29), we realize that the potential parameters for $\mathcal{E}_{\nu}=-5$ Ry apply to the 15 largest $\vec{G}_{11}$ 's with an accuracy better than $0.2 \mathrm{mRy}$. The only noticeable change of the energies in Table II is that of the highest energy $[E-v(-\infty) \approx 0.90-1.07 \mathrm{Ry}=-0.17$ Ry] which becomes less accurate by $4 \mathrm{mRy}$ as $\mathcal{E}_{\nu}$ corresponding to $\vec{G}_{\|}=(0,0)$ is changed from -0.5 to $-1.0 \mathrm{Ry}$. The values of $\left\langle\dot{\phi}_{\nu}^{2}\right\rangle^{-1 / 2}$ tend towards

TABLE II. Dependence of the energies for the (100) monolayer of $\mathrm{Cu}$ of the choice of $\mid \mathcal{E}_{\nu}\left(\overrightarrow{\mathrm{G}}_{\| 1}\right)$ [as in Eq.(21)] for the planar muffin tins.

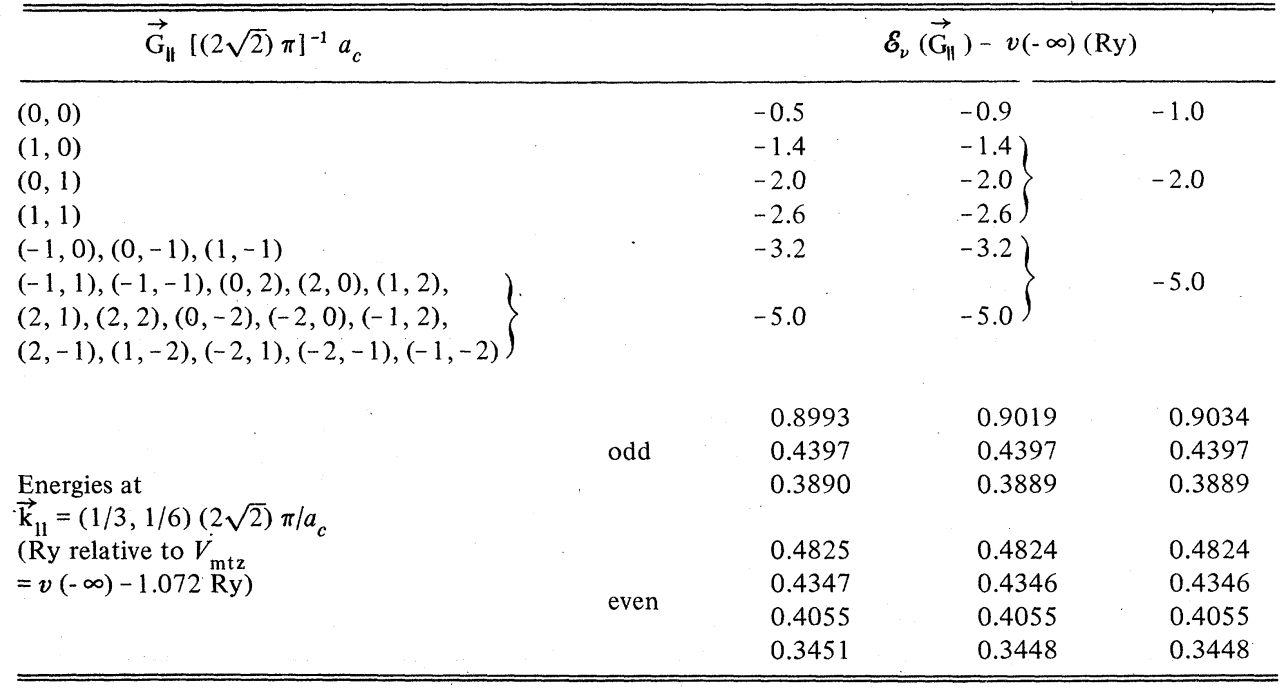


zero for $\mathcal{E}_{\nu}$ values increasing towards $v(-\infty)$ (see Appendix B) and, for $\mathcal{E}_{\nu}=-0.5$ and $-1.0 \mathrm{Ry}$, we find $\left\langle\dot{\phi}_{v}^{2}\right\rangle^{-1 / 2}=1.29$ and $3.03 \mathrm{Ry}$, respectively. If one chooses to let $\mathcal{E}_{\nu}$ be independent of $\overrightarrow{\mathrm{k}}_{\| 1}$, it is therefore impossible to use any $\mathcal{E}_{\nu}$ higher than about -0.5 Ry because $\max \left(k_{\|}^{2}\right)$ is about $1 \mathrm{Ry}$. The example in Table II indicates that even with a maximum $\mathcal{E}_{\nu}$ of $-1 \mathrm{Ry}$, the errors remain small. In conclusion, we found that three sets of $\vec{k}_{11}$-independent potential parameters for the planar regions were sufficient to yield an accuracy better than 5 mRy.

The energy bands shown in Figs. 4 and 5 along the symmetry lines of the Brillouin zones for the (100) and (111) monolayers, consist of five narrow $d$ bands crossed by and hybridizing with a broad $s p$ band. When comparing our energy bands for the (100) layer with those of Kar and Soven ${ }^{15}$ we find that the structures along the line from $\Gamma$ to $M$ are identical. The $d$-band widths are slightly different, but no more than one would expect from the differences in the potential constructions.

The densities of states for the (100) and (111) band structures are shown in Figs. 6 and 7 . They consist of a relatively small $s p$ contribution with the shape of a step function and a large $d$ contribution with pronounced structure. The $d$-band widths for the two films are $W_{d}^{100}=0.15 \mathrm{Ry}$ and $W_{d}^{111}=0.20 \mathrm{Ry}$ while, for the bulk, $W_{d}=0.25 \mathrm{Ry}$. The ratios $W_{d}^{100} / W_{d}=0.60$ and $W_{d}^{111} / W_{d}=0.80$ are nearly equal to the square root of the ratios between the numbers of nearest neighbors [i.e., $\left(\frac{4}{12}\right)^{1 / 2}=0.58$ and $\left(\frac{6}{12}\right)^{1 / 2}=0.71$, respectively]. This is what one would expect from a simple tight-binding theory with constant transfer integrals [see, for instance, (LM 4.34)].

The densities of states were computed with a linear interpolation technique described in Appendix A. We used a uniform $\vec{k}_{11}$ mesh of size $\frac{1}{12} \Gamma X$ and $\frac{1}{12} \Gamma M$ for the (100) and (111) films, re-

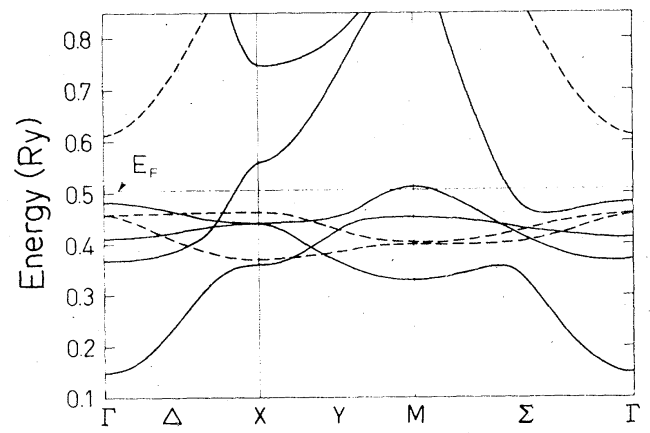

FIG. 4. Band structure along the symmetry lines of the two-dimensional Brillouin zone for the (100) monolayer of $\mathrm{Cu}$. The states even (odd) under reflection are drawn in full (dashed) lines. The zero of energy is at $V_{\mathrm{mtz}}$ $=v(-\infty)-1.072 \mathrm{Ry}$. spectively. This gives 91 points in the $2 \mathrm{D}-\mathrm{IBZ}$, including 36 points on symmetry lines, and thus divides the irreducible zone into 144 triangular microzones. These are the regions over which the energy bands are linearly interpolated.

It turned out that the computational speed of our method is essentially determined by the time it takes to solve the eigenvalue problem (2).

\section{CONCLUSION}

We have generalized the linear APW energyband method to deal with thin films. The method has the advantages that it is computationally efficient, it can treat a general local potential, it can be made self-consistent, and it applies to simple as well as transition metals.

In order to illustrate and test the method, we have presented the results of energy band and density of states calculations for (100) and (111) monolayers of $\mathrm{Cu}$ using a muffin-tin potential, and we find good agreement with the results of Kar and Soven. ${ }^{15}$

\section{APPENDIX A}

In order to calculate spectral properties of films (e.g., densities of states, optical transitions, etc.) we have adapted the tetrahedron method for three-dimensional $\overrightarrow{\mathrm{k}}$-space integration $\mathrm{s}^{18}$ to two dimensions.

For simplicity we shall only consider the calculation of the density of states

$$
\begin{aligned}
N(E)= & 2 A(2 \pi)^{-2} \\
& \times \sum_{j} \int_{E_{j}\left(\overrightarrow{\mathrm{k}}_{\|}\right)=E}\left|\nabla_{\overrightarrow{\mathrm{k}}_{\| 1}} E_{j}\left(\overrightarrow{\mathrm{k}}_{\| 1}\right)\right|^{-1} d k_{\|} .
\end{aligned}
$$

The 2D-BZ is divided into triangles of equal

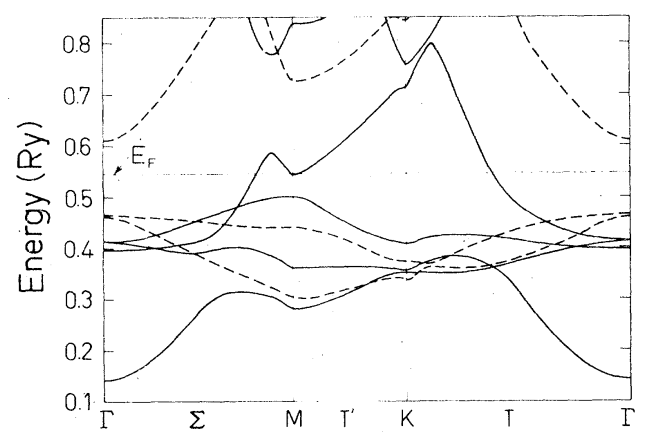

FIG. 5. Band structure along the symmetry lines of the two-dimensional Brillouin zone for the (111) monolayer of $\mathrm{Cu}$. The states even (odd) under reflection are drawn in full (dashed) lines. The zero of energy is at $V_{\mathrm{mtz}}$ $=v(-\infty)-1.072 \mathrm{Ry}$. 


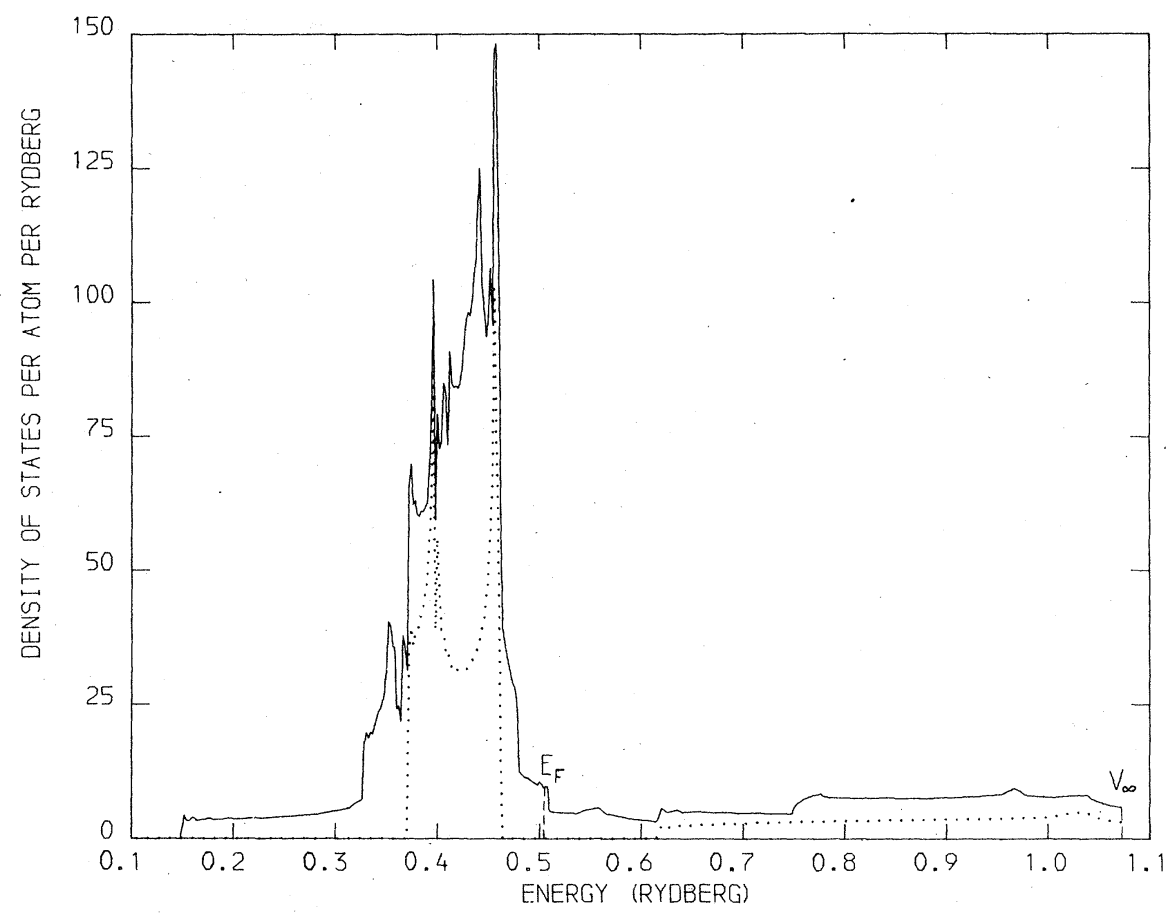

FIG. 6. Density of states for the $(100)$ monolayer of $\mathrm{Cu}$. The contribution from the odd states is indicated by the dotted line. areas. In each triangle the energy band $E_{j}\left(\overrightarrow{\mathrm{k}}_{11}\right)$ is approximated by the linear function which takes the proper values in the corners of the triangle, and the integral (A1) is replaced by

$$
\begin{aligned}
N(E) & \approx 2 A(2 \pi)^{-2} \sum_{j, i}|\nabla E|_{j i}^{-1} l_{j i}(E) \\
& \equiv \sum_{j, i} N_{j i}(E)
\end{aligned}
$$

Here $l_{j i}(E)$ is the length within the $i$ th triangle of the constant-energy line of energy $E,|\nabla E|_{j i}$ is the magnitude of the gradient in the triangle, and $j$ is the band index.
We shall now derive the expressions for $N_{j i}(E)$ in terms of the three energies $E_{1} \leqslant E_{2} \leqslant E_{3}$ at the corners of the $i$ th triangle. We drop the subscripts $j$ and $i$.

Since $l(E)$ is a continuous and piecewise linear function of energy we obtain, for the interval $E_{1} \leqslant E \leqslant E_{2}$,

$$
\begin{aligned}
& N(E)=4 I^{-1} \frac{E-E_{1}}{\left(E_{2}-E_{1}\right)\left(E_{3}-E_{1}\right)}, \\
& n(E)=2 I^{-1} \frac{\left(E-E_{1}\right)^{2}}{\left(E_{2}-E_{1}\right)\left(E_{3}-E_{1}\right)},
\end{aligned}
$$

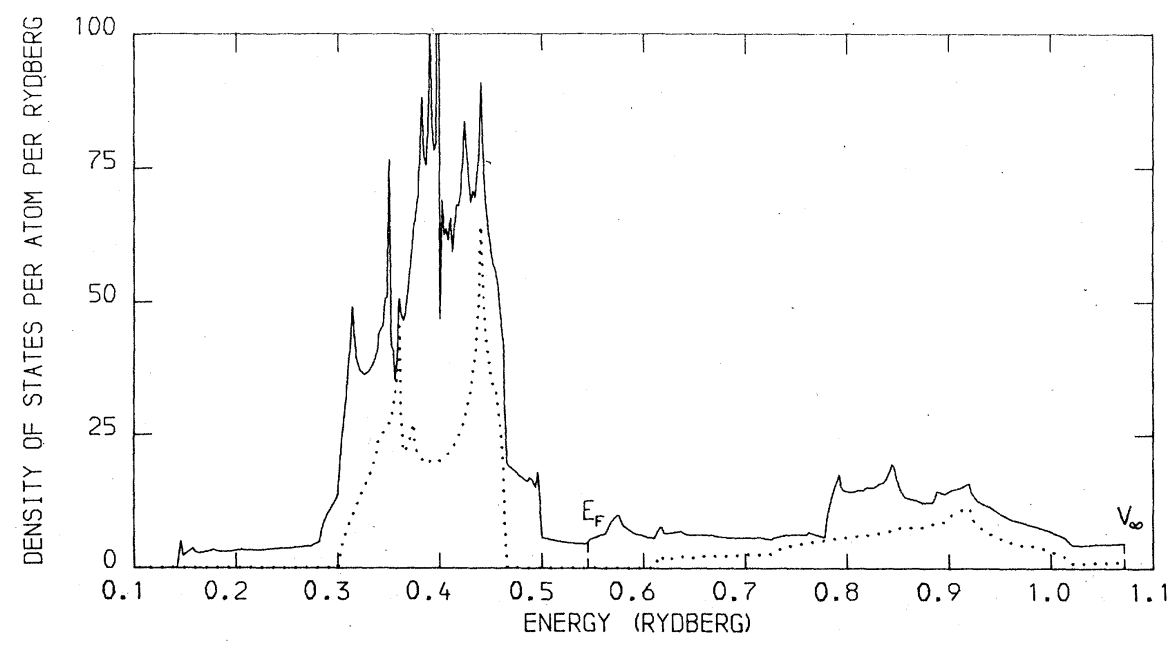

FIG. 7. Density of states for the (111) monolayer of $\mathrm{Cu}$. The contribution from the odd states is indicated by the dotted line. 
TABLE III. Vacuum potential parameters for a constant (zero) potential.

$$
\begin{aligned}
& \dot{D}_{\nu}=\left(-\mathcal{E}_{\nu}\right)^{\frac{1}{2}} \\
& D_{\nu}^{1}=3\left(-\mathcal{E}_{\nu}\right)^{\frac{1}{2}} \\
& \phi_{\nu}^{2}=2\left(-\mathcal{E}_{\nu}\right)^{\frac{1}{2}} \\
& \left\langle\dot{\phi}_{\nu}^{2}\right\rangle^{-\frac{1}{2}}=-4 \mathcal{E}_{\nu}
\end{aligned}
$$

and, for the interval $E_{2} \leqslant E \leqslant E_{3}$,

$$
\begin{aligned}
& N(E)=4 I^{-1} \frac{E_{3}-E}{\left(E_{3}-E_{1}\right)\left(E_{3}-E_{2}\right)}, \\
& n(E)=2 I^{-1}\left[1-\frac{\left(E-E_{3}\right)^{2}}{\left(E_{3}-E_{2}\right)\left(E_{3}-E_{1}\right)}\right] .
\end{aligned}
$$

Here, $I$ is the number of triangles in the 2D-IBZ and $n(E)$ is the integrated density of states which, for $E \leqslant E_{1}$, equals zero and, for $E \geqslant E_{3}$, equals $2 I^{-1}$. For $E \leqslant E_{1}$ or $E \geqslant E_{3}, N(E)$ equals zero.

\section{APPENDIX B}

We shall give the vacuum-potential parameters for the case that the potential is constant in the vacuum region $(q \leqslant 0)$. These parameters may serve as a reference for vacuum-potential parameters in general.

For

$$
v(q)=0 \text { and } \mathcal{E} \equiv-\lambda^{2}<0,
$$

the normalized solution of (18) is

$$
\phi(\mathscr{E}, q)=(2 \lambda)^{1 / 2} e^{\lambda q}
$$

and, by differentiation with respect to $\mathcal{E}$,

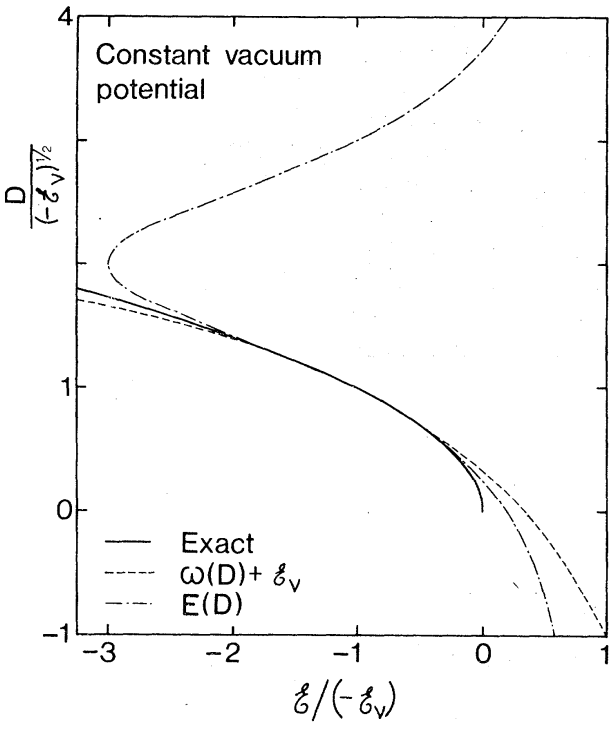

FIG. 8. Exact logarithmic derivative, $D(\mathcal{E})=(-\mathcal{E})^{1 / 2}$, and the estimates (25) and (29) for a vacuum region of zero potential.

$$
\dot{\phi}(\mathcal{E}, q)=-(2 \lambda)^{-3 / 2}(1+2 \lambda q) e^{\lambda q} .
$$

The logarithmic derivatives are

$$
D(\mathcal{E})=\phi^{\prime}(\mathcal{E}, 0) / \phi(\mathcal{E}, 0)=\lambda,
$$

and

$$
D^{1}(\mathcal{E})=\dot{\phi}^{\prime}(\mathcal{E}, 0) / \dot{\phi}(\mathcal{E}, 0)=3 \lambda .
$$

The potential parameters for an arbitrary $\mathcal{E}_{\nu}$ are given in Table III. In Fig. 8 we show the exact logarithmic derivative together with the estimates (25) and (29) which have errors of order $\left(\mathcal{E}-\mathcal{E}_{\nu}\right)^{3}$ and $\left(\mathscr{E}-\mathcal{E}_{\nu}\right)^{4}$, respectively.
${ }^{1}$ P. Hohenberg and W. Kohn, Phys. Rev. 136, B 864 (1964); W. Kohn and L. J. Sham, ibid. 140, A1133 (1965).

${ }^{2}$ O. K. Andersen, Phys. Rev. B 12, 3060 (1975).

${ }^{3}$ D. D. Koelling and G. O. Arbman, J. Phys. F $\underline{5}, 2041$ (1975).

${ }^{4}$ J. E. Müller, O. Jepsen, O. K. Andersen, and J. W. Wilkins, Phys. Rev. Lett. 40, 720 (1978); and (unpublished).

${ }^{5} \mathrm{~J}$. F. Janak and A. R. Williams, Phys. Rev. B 14, 4199 (1976); V. L. Moruzzi, A. R. Williams, and J. F. Janak, ibid. 15, 2854 (1977).

${ }^{6}$ U. K. Poulsen, J. Kollár, and O. K. Andersen, J. Phys. F $\underline{6}$, L241 (1976); O. K. Andersen, J. Madsen, U. K. Poulsen, O. Jepsen, and J. Kollár, Physica (Utr.) 86-88B, 249 (1977).

${ }^{7}$ O. Gunnarsson, J. Harris, and R. O. Jones, Phys. Rev. B 15, 3027 (1977); O. Gunnarsson, J. Harris, and R. O. Jones, J. Chem. Phys. 67,3970 (1977).
${ }^{8} \mathrm{~J}$. Harris, R. O. Jones, and O. Gunnarsson, J. Chem. Phys. (1978).

${ }^{9}$ L. Hedin and B. I. Lundquist, J. Phys. C 4 2064 (1971); U. von Barth and L. Hedin, ibid. $\underline{5}, 1629$ (1972); O. Gunnarsson and B. I. Lundquist, Phys. Rev B $\underline{13}$, 4274 (1976).

${ }^{10}$ O. K. Andersen and R. G. Woolley, Mol. Phys. 26, 905 (1973).

${ }^{11}$ R. V. Kasowski, Phys. Rev. Lett. $\underline{33}, 83$ (1974); 33 , 1147 (1974); Solid State Commun. 17, 179 (1975); Phys. Rev. B 14, 3398 (1976).

${ }^{12}$ H. Krakauer and B. R. Cooper, Phys. Rev. B 16, 605 (1977); J. Madsen and O. K. Andersen (unpublished).

${ }^{13}$ O. K. Andersen and O. Jepsen, Physica (Utr.) B + C91, 317 (1977); O. K. Andersen, W. Klose, and H. Nohl, Phys. Rev. B. 17, 1209 (1978).

${ }^{14}$ K. S. Sohn, D. G. Dempsey, L. Kleinman, and E. Caruthers, Phys. Rev. B 13, 1515 (1976), and references therein. 
${ }^{15}$ W. Kohn, Phys. Rev. B 11, 3756 (1975); N. Kar and P. Soven, ibid. 11, 3761 (1975).

${ }^{16}$ S. G. Louie, K.-M. Ho, J. R. Chelikowsky, and M. L. Cohen, Phys. Rev. Lett. 37, 1289 (1976); and Phys. Rev. B 15, 5627 (1977).

${ }^{17}$ J. G. Gay, J. R. Smith, and F. J. Arlinghaus, Phys. Rev: Lett. 38, 561 (1977).

${ }^{18}$ O. Jepsen and O. K. Andersen, Solid State Commun. $\underline{9}$, 1763 (1971); G. Lehman and M. Taut, Phys. Status Solidi B 54, 469 (1972).
${ }^{19} \mathrm{~J}$. Madsen and O. K. Andersen (unpublished).

${ }^{20}$ L. F. Mattheiss, Phys. Rev. 133, A1399 (1964).

${ }^{21}$ R. M. Nieminen and C. H. Hodges, J. Phys. F 6,573 (1976).

${ }^{22}$ Although Gay et al. (Ref. 17) did not include the 4p orbitals in their LCAO calculation, the plot of the charge density outside the surface seems to indicate that, with $a-b=s$, the non-muffin-tin correction to the potential can be neglected in region IV. 